\title{
The impact of ColRS two-component system and TtgABC efflux pump on phenol tolerance of Pseudomonas putida becomes evident only in growing bacteria
}

\author{
Marta Putrinš, Heili Ilves, Liisa Lilje, Maia Kivisaar and Rita Hõrak*
}

\begin{abstract}
Background: We have recently found that Pseudomonas putida deficient in CoIRS two-component system is sensitive to phenol and displays a serious defect on solid glucose medium where subpopulation of bacteria lyses. The latter phenotype is significantly enhanced by the presence of phenol in growth medium. Here, we focused on identification of factors affecting phenol tolerance of the colR-deficient $P$. putida.

Results: By using transposon mutagenesis approach we identified a set of phenol-tolerant derivatives of colR-deficient strain. Surprisingly, half of independent phenol tolerant clones possessed miniTn5 insertion in the $\operatorname{tg} A B C$ operon. However, though inactivation of TtgABC efflux pump significantly enhanced phenol tolerance, it did not affect phenolenhanced autolysis of the colR mutant on glucose medium indicating that phenol- and glucose-caused stresses experienced by the colR-deficient P. putida are not coupled. Inactivation of $\operatorname{Ttg} A B C$ pump significantly increased the phenol tolerance of the wild-type $P$. putida as well. Comparison of phenol tolerance of growing versus starving bacteria revealed that both CoIRS and TtgABC systems affect phenol tolerance only under growth conditions and not under starvation. Flow cytometry analysis showed that phenol strongly inhibited cell division and to some extent also caused cell membrane permeabilization to propidium iodide. Single cell analysis of populations of the $\operatorname{tg} C$ - and colRttgCdeficient strains revealed that their membrane permeabilization by phenol resembles that of the wild-type and the colR mutant, respectively. However, cell division of P. putida with inactivated TtgABC pump seemed to be less sensitive to phenol than that of the parental strain. At the same time, cell division appeared to be more inhibited in the colRmutant strain than in the wild-type P. putida.
\end{abstract}

Conclusions: ColRS signal system and TtgABC efflux pump are involved in the phenol tolerance of P. putida. However, as they affect phenol tolerance of growing bacteria only, this indicates that they participate in the regulation of processes which are active during the growth and/or cell division. Single cell analysis data indicated that the cell division step of cell cycle is particularly sensitive to the toxic effect of phenol and its inhibition can be considered as an adaptive response under conditions of phenol stress.

\section{Background}

Aromatic compounds in the environment can either be plant-derived or released as a result of human action. Phenol and other aromatics can be highly toxic, yet their toxicity depends on the concentration of the compound as well as on tolerance level of bacteria. Aromatics such

\footnotetext{
* Correspondence: rhorak@ebc.ee

${ }^{1}$ Institute of Molecular and Cell Biology, University of Tartu, 51010 Tartu, Estonia
}

Full list of author information is available at the end of the article as toluene, xylenes and phenol are harmful, because they dissolve easily in cell membrane, disorganizing its structure and impairing vital functions [1-3]. Disruption of membrane integrity affects crucial membrane functions like acting as a barrier, energy transducer and matrix for enzymes and to certain extent, it also affects cell division and DNA replication. Chaotropic solutes like phenol can also weaken electrostatic interactions in and between biological macromolecules and influence water availability without remarkably affecting cell turgor [4]. When 
encountering a hazardous aromatic compound, several adaptive responses are triggered in bacteria to neutralize the action of a toxicant. For instance, organic solvent tolerance of $P$. putida relies on several concurrently acting processes: repulsion of solvent molecules, restructuring of cell membrane to reduce harmful effects of the solvent, and active efflux of solvent from the cell $[2,5]$.

Bacterial cell membrane is not only the first target of environmental stress but in many cases it acts also as the first sensor triggering a stress response. The stress signal can emerge from changed membrane properties or from specific signal molecule recognised by a membraneembedded sensor protein. The ability of bacteria to monitor changes in the environment and to adjust their gene expression accordingly vastly depends on functioning of two-component signal transduction systems (TCS) [6]. TCSs are typically composed of a membrane-located sensor with histidine kinase activity and of a cytoplasmic response protein with a signal-accepting receiver domain. Environmental signal sensed by membrane protein is transduced to a response regulator by phosphorylation.

Bacteria from Pseudomonas genus possess tens of different two-component systems. Genes coding for ColRS signal system are conserved in all so far sequenced Pseudomonas species http://www.pseudomonas.com indicating its importance in different habitats and environmental conditions. ColRS system was first described in P. fluorescens due to its ability to facilitate root colonization by this bacterium [7]. Our studies with P. putida have revealed involvement of ColRS TCS in several unrelated phenotypes. First, disruption of ColR response regulator gene resulted in lowered phenol tolerance of $P$. putida [8]. Second, different mutational processes such as point mutations and transposition of $\operatorname{Tn} 4652$ were repressed in starving colS- and colR-knockout $P$. putida $[8,9]$. We associated the latter phenotype with phenol tolerance as the mutation frequency in a colR-deficient strain, in contrast to the wild-type, depended on phenol concentration in selective medium [8]. Third, cell population of colR-deficient $P$. putida growing on glucose solid medium was heterogeneous: a distinct subpopulation of cells possessed a propidium iodide-permeable cell membrane and a fraction of cells underwent lysis [10]. It is notable that glucose-dependent cell lysis of colR-mutant was significantly enhanced if phenol was present in the growth medium [10].

Identification of ColRS-regulated genes has pointed to cell membrane as a potential target of this particular TCS. Namely, the operon locating just downstream of colRS genes that codes for a probable lipopolysaccharide kinase and a methyltransferase is positively controlled by ColR both in P. putida and P. fluorescens $[11,12]$. In addition, $P$. putida ColRS system negatively regulates transcription of opr $Q$ and $\operatorname{alg} D$ genes that code for outer membrane porin and alginate biosynthesis enzyme, respectively [8]. Genome-wide search for ColR regulon in P. putida has revealed several other ColR-regulated membrane proteins such as lipid A 3-O-deacylase PagL and diacylglycerol kinase DgkA involved in metabolism of lipopolysaccharides and phospholipides, respectively [12]. Importantly, the presence of phenol in growth medium significantly enhances the effect of ColR on its target promoters $[8,12]$ pointing once more to increased phenol sensitivity of the colR mutant $P$. putida.

Many ColR-regulated genes have been tested with respect to their potential participation in the phenol tolerance of $P$. putida. However, despite several efforts we could not identify so far any particular ColR target gene responsible for reduced phenol tolerance of the colR-deficient $P$. putida (our unpublished data). Here, to further unravel the role of ColRS system in phenol tolerance, we report on a transposon mutagenesis performed in a colRdeficient strain to search for suppressors of phenol sensitivity. This screen disclosed several genes, disruption of which enhanced phenol tolerance of the colR mutant. Additionally, we show that phenol sensitivity of the colRdeficient bacteria becomes evident only under growthpermitting conditions and not if bacteria are starving for a carbon source. Population analysis at single cell level indicated that particularly cell division is inhibited under condition of phenol stress.

\section{Methods}

\section{Bacterial strains and media}

All strains used in this study are derivatives of $P$. putida PaW85 [13], which is isogenic to fully sequenced KT2440 [14]. To study the role of ColRS system, previously constructed colR- and colS-knockout derivatives of P. putida PaW85, PaWcolR and PaWcolS [9] were exploited. Escherichia coli strains DH5 $\alpha$ [15] and CC118 $\lambda$ pir [16] were used for DNA cloning procedures, and HB101 [17] as a host for helper plasmid pRK2013 [18]. E. coli was grown at $37^{\circ} \mathrm{C}$ and $P$. putida at $30^{\circ} \mathrm{C}$. Bacteria were grown in Luria-Bertani (LB) medium [19] or in M9 minimal medium [20] containing either $10 \mathrm{mM}$ glucose or $10 \mathrm{mM}$ gluconate. Phenol concentrations in minimal media are specified in the text, as they varied between the experiments. When selection was necessary, antibiotics were added at following final concentrations: ampicillin (100 $\left.\mu \mathrm{g} \mathrm{ml}^{-1}\right)$, kanamycin $\left(50 \mu \mathrm{g} \mathrm{ml}^{-1}\right)$, or streptomycin $(20 \mu \mathrm{g}$ $\left.\mathrm{ml}^{-1}\right)$ for $E$. coli and bezylpenicillin $\left(1500 \mu \mathrm{g} \mathrm{ml}^{-1}\right)$, kanamycin $\left(50 \mu \mathrm{g} \mathrm{ml}^{-1}\right)$, or streptomycin $\left(200 \mu \mathrm{g} \mathrm{ml}^{-1}\right)$ for $P$. putida.

\section{Selection strategy of phenol tolerant mutants in colR- deficient $P$. putida strain}

For identification of genes affecting phenol sensitivity, the colR-deficient strain was subjected to mutagenesis by $\operatorname{Tn} 5$ 
based mini-transposon containing streptomycin resistance marker. A mini-transposon-carrying plasmid mTn5SSgusA40 [21] was conjugatively transferred from E. coli CC118 Apir [16] into a P. putida colR-deficient strain with the aid of a helper plasmid pRK2013 [18]. Transconjugants with random chromosomal insertions of the mini-transposon were first selected on glucose minimal plates supplemented with kanamycin and streptomycin. After colonies were grown for three days at $30^{\circ} \mathrm{C}$, they were replicated onto glucose minimal plates containing $8 \mathrm{mM}$ phenol. Although a single colR-deficient cell could not form a colony on these plates, replication of big and closely located colonies of colR-deficient bacteria enabled their growth on replica plates. After another three days, growth of replicated clones in the presence of phenol was evaluated. About 150 transconjugants out of approximately 9000 transposon mutants grew better than colR-deficient P. putida and they were subjected to secondary assay of phenol tolerance. In order to avoid spontaneous phenol tolerant mutants, the clones of interest were picked up from glucose plates of initial selection. The secondary screen yielded 34 clones with higher phenol tolerance than the parental colR-deficient strain. Finally, siblings were eliminated through analysis of clones by arbitrary PCR and sequencing, resulting in 27 independent transposon insertion mutants with elevated phenol tolerance.

\section{Arbitrary PCR}

To identify chromosomal loci interrupted by insertion of mini-transposon in selected clones arbitrary PCR and sequencing were used. PCR products were generated by two rounds of amplifications as described elsewhere [22]. In the first round, a primer specific for the Sm gene (Smsaba - 5'-GAAGTAATCGCAACATCCGC-3') or for the gusA gene (Gus2 - 5'-ACTGATCGTTAAAACTGCCTGG) and an arbitrary primer were used (Arb6 - 5'GGCCACGCGTCGACTAGTACNNNNNNNNN-

NACGCC-3'). Second-round PCR was performed with primers Smsaba or Gus2 and Arb2 (5'-GGCCACGCGTCGACTAGTAC-3').

\section{Cloning procedures and construction of bacterial strains}

To inactivate the $\operatorname{tg} C$ gene in both wild-type and colRdeficient backgrounds the $\operatorname{tg} C$ gene was first amplified using oligonucleotides ttgCalgus (5'-GAAGAATTCGTCACCCCTGAAAATCC-3') and ttgClopp (5'-CCGAATTCGGTGGGCTTTCTGCTTTT-3') and inserted into EcoRI-opened pUCNotKm (R. Teras). For disruption of the $t \operatorname{tgC}$ gene in pUC/ttgC, a central 315-bp Eco255I fragment of $t \operatorname{tgC}$ was replaced with $\mathrm{Sm}^{\mathrm{r}}$ gene from the pUTmini-Tn5Sm/Sp [23]. The resulting $\operatorname{ttgC::Sm~sequence~}$ was cloned from pUC/ttgC::Sm into pGP704L as a EcoRI fragment [24]. Plasmid pGP704L/ttgC::Sm was selected in $E$. coli strain CC118 $\lambda$ pir and the interrupted $\operatorname{tg} C$ gene was inserted into the chromosome of P. putida PaW85 and PaWcolR by homologous recombination.

For disruption of the $\operatorname{tg} B$, the $5^{\prime}$ end of the gene was amplified with oligonucleotides ttgBXba (5'-CAATCTAGAACTGCGCCAGCTCAAGGC) and ttgBSac (5'CCCGAGCTCTGTTCCATCGAGCGTTTG) and cloned into Eco32I-opened pBluescript KS (Stratagene). The cloned $\operatorname{tg} B$ sequence was disrupted by replacing of a central 735-bp EheI fragment with $\mathrm{Sm}^{\mathrm{r}}$ gene and the resulting $\operatorname{tg} B:: S m$ sequence was subcloned into pGP704L as a XbaI-SacI fragment. Finally, the interrupted $\operatorname{tg} B$ gene was inserted into the chromosome of P. putida PaW85 and PaWcolR by homologous recombination.

\section{Measurement of unmasked $\beta$-galactosidase activity}

$\beta$-galactosidase activities were measured from solid medium-grown bacteria. As a source of $\beta$-galactosidase, the plasmid pKTlacZS/C containing the $\operatorname{tnp} A$ promoter of the transposon $\operatorname{Tn} 4652$ in front of the lac $Z$ gene, was used [25]. Bacteria grown overnight on solid glucose M9 minimal medium or on the same medium supplemented with $1 \mathrm{mM}$ phenol were scraped off from the plates using plastic swabs. Cells were suspended in M9 solution and optical density of the suspension was determined at OD580. $\beta$-galactosidase activity was measured using two alternative procedures. In one procedure, SDS and chloroform were added to the reaction to permeabilize bacterial cell membrane as described previously [26]. In a parallel experiment SDS and chloroform were not added. Percentage of unmasked $\beta$-galactosidase activity was calculated by equation: $\mathrm{xn} / \mathrm{xp} \times 100 \%$, where $\mathrm{xp}$ is $\beta$-galactosidase activity measured in assay with SDS and chloroform, and $\mathrm{xn}$ is $\beta$-galactosidase activity measured using non-permeabilized cells.

\section{Phenol tolerance assay on solid medium}

Phenol sensitivity was evaluated on agar plates containing $10 \mathrm{mM}$ glucose or $10 \mathrm{mM}$ gluconate as carbon sources, and up to $10 \mathrm{mM}$ phenol (specified in the Fig. 1). Approximately $1 \times 10^{5}$ cells were spotted onto plates as $5 \mu$ l drops and plates were incubated at $30^{\circ} \mathrm{C}$ for 48 hours.

\section{Phenol mediated killing assay}

Bacteria were pre-grown on solid glucose minimal plates for 24 hours. Cells were scraped off from the plates and suspended in M9 buffer containing $10 \mathrm{mM}$ glucose and microelements. Optical density of cell suspension was adapted to 0.2 at $\mathrm{OD}_{580}$. A number of colony forming units (CFU) was determined directly before and 10, 20 and 30 minutes after addition of $50 \mathrm{mM}$ phenol. Experiment was carried out at $30^{\circ} \mathrm{C}$. 


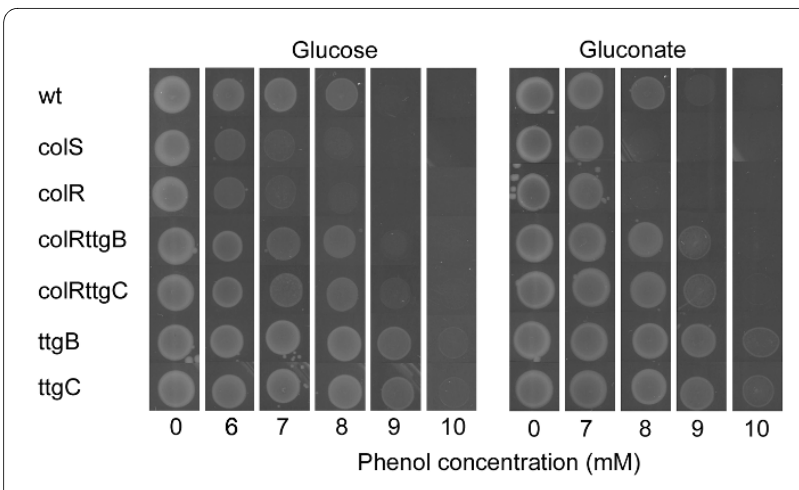

Figure 1 Plate assay of phenol tolerance. Results of P. putida PaW85 (wt), cols-deficient (colS), colR-deficient (colR), ttgB-deficient (ttgB), ttgC-deficient (ttgC), colRttgB double mutant (colRttgB) and colRttgC double mutant (colRttgC) strains are presented. Approximately $1 \times 10^{5}$ cells were spotted onto solid medium and plates were incubated at $30^{\circ} \mathrm{C}$ for 48 hours. The minimal media contained either $10 \mathrm{mM}$ glucose or $10 \mathrm{mM}$ gluconate as the carbon source. Concentration of added phenol is indicated below the figures.

\section{Phenol tolerance microtiter plate assay}

Phenol sensitivity was evaluated on microtiter plates containing $100 \mu \mathrm{l} 9$ minimal medium in the presence of 10 $\mathrm{mM}$ glucose or $10 \mathrm{mM}$ gluconate or in the absence of carbon source. LB-grown overnight cultures were diluted into M9 solution and kept without carbon source for two hours to allow using up any residual carbon and energy source from medium. After that about $5 \times 10^{5}$ cells per ml were inoculated into the microtiter plates containing different phenol concentrations and appropriate carbon source (if added at all). Microtiter plates were incubated at $30^{\circ} \mathrm{C}$ with shaking and after 24 hours the CFU was assessed.

\section{Flow cytometry analysis}

$P$. putida cells, grown for $24 \mathrm{~h}$ on glucose or gluconate minimal plates with different concentration of phenol, were stained using the LIVE/DEAD BacLight kit (Invitrogen). The kit contains a red fluorescence dye propidium iodide (PI) and green fluorescence dye SYTO9, which both stain nucleic acids. The SYTO9 is able to penetrate all cells, whereas PI enters only the cells with damaged cytoplasmic membranes. If the two dyes are combined then the emission properties of the stain mixture bound to DNA change due to displacement of one stain by the other and quenching by fluorescence resonance energy transfer [27]. Thus, decreased green fluorescence of SYTO9 in the presence of PI indicates entrance of PI into the cells. Staining of cells was performed as suggested by manufacturers and approximately 10000 events from every sample were analysed with flow cytometer FACSAria (BD Biosciences). Excitation of fluorescent dyes was performed using $488 \mathrm{~nm}$ laser. Forward and side scatter (FCS and SSC, respectively) of the light and fluo- rescence emission at 530 (30) and 616 (26) were acquired for every event. To calculate significance of differences of subpopulations between two strains the Students T-test was performed. Probability was calculated using twosample equal variance type of T-test and two-tailed distribution.

\section{Results}

Inactivation of different genes involved in membrane, central metabolism or regulatory functions can increase phenol tolerance of colR-deficient strain

The growth of colR and colS mutant cells is precluded on glucose and gluconate solid medium in the presence of 8 $\mathrm{mM}$ phenol, while the growth of the wild-type is not [8] (Fig. 1). However, after few days of incubation of a colRdeficient strain on phenol-containing plates, the phenol tolerant mutants appeared with high frequency, approximately $10^{-4}$ mutants per cell inoculated (Additional File 1). The high frequency of suppression of phenol sensitivity of colR mutant encouraged us to apply transposon mutagenesis for identification of genes implicated in phenol tolerance and potentially interfering in ColRS pathway.

For identification of genes affecting phenol sensitivity, the colR-deficient strain was subjected to transposon mutagenesis. Screening of about 9000 transposon insertion derivatives of the colR mutant disclosed 27 clones with higher phenol tolerance. Sequencing of mini-transposon insertion sites revealed that phenol sensitivity of the colR-deficient strain was elevated by disruption of genes dispersed between different functional classes (Table 1). As ColRS system is obviously involved in membrane functionality $[8,11,12]$ it was expected that disruption of several membrane-related genes could complement the colR-deficiency. However, some metabolic genes were also identified as determinants of phenol tolerance (Table 1). Most of mini-transposon insertions were located in open reading frames of targeted genes, thus obviously abolishing their function. However, in case of PP1824 the mini-transposon was inserted upstream of the ATG start codon most probably changing the expression level of this gene.

\section{Disruption of $\mathrm{ttgC}$ enhances phenol tolerance of both colR- deficient and colR-proficient P. putida}

14 out of 27 phenol tolerant minitransposon derivatives of the colR-deficient strain possessed miniTn5 insertion in the $\operatorname{tg} A B C$ operon (Table 1) and therefore we focused on this system. In toluene tolerant Pseudomonas putida DOT-T1E, three homologous efflux pumps TtgABC, TtgDEF and TtgGHI belonging to the RND (resistancenodulation-cell division) family transporters contribute to solvent tolerance [28]. TtgABC efflux pump plays a major role in antibiotic resistance of this strain, and it 
Table 1: Description of chromosomal loci of phenol tolerant mini-transposon derivatives of colR-deficient $P$. putida http:// www.jcvi.org/.

\begin{tabular}{llllc}
\hline Locus ID & Gene name & Protein name & $\begin{array}{l}\text { Probable } \\
\text { localization* }\end{array}$ & $\begin{array}{l}\text { Number of } \\
\text { Insertions }\end{array}$ \\
\hline PP0145 & & Nat/Pi cotransporter family protein & $\mathrm{CM}$ & 1 \\
PP1386 & $\operatorname{tgA}$ & multidrug/solvent RND membrane fusion protein & $\mathrm{CM}$ & 4 \\
PP1385 & $\operatorname{tgB}$ & multidrug/solvent RND transporter & $\mathrm{CM}$ & 9 \\
PP1384 & $\operatorname{tgC}$ & multidrug/solvent RND outer membrane protein & $\mathrm{OM}$ & 1 \\
PP1619 & & conserved hypothetical protein & $\mathrm{C}$ & 1 \\
PP1621 & pcm & protein-L-isoaspartate O-methyltransferase & $\mathrm{C}$ & 4 \\
PP1650 & gacS & sensor histidine kinase-response regulator & $\mathrm{CM}$ & 3 \\
PP1842 & & glutamine amidotransferase, class I & $\mathrm{C}$ & $1^{* *}$ \\
PP3997 & & glycosyl transferase, putative & $\mathrm{C}$ & 1 \\
PP4422 & & succinate-semialdehyde dehydrogenase, putative & $\mathrm{C}$ & 1 \\
PP4798 & & membrane-bound lytic murein transglycosylase, putative & $\mathrm{CM}$ & 1 \\
\hline
\end{tabular}

* Abbreviations: CM - cytoplasmic membrane; OM - outer membrane; C - cytoplasm

** insertion 3 bp upstream of ATG

also expels solvents and plant antimicrobials from cells [28-31]. The basal expression level of TtgABC in Pseudomonas putida DOT-T1E is relatively high being further enhanced by hydrophobic antibiotics and some plant metabolites [30,31]. However, the expression of this efflux system does not respond to solvents [29]. TtgABC efflux pump proteins are highly similar between DOTT1E and KT2440 strains (over 99\% identity) suggesting that their substrate range and biological role could be similar. To find out whether inactivation of $\operatorname{tg} A B C$ operon only complements the defect of a colR-mutant or it can also influence phenol tolerance of the wild-type strain, the $\operatorname{tg} A B C$ operon was disrupted in the colR as well as in the wild-type backgrounds.

In addition to phenol stress, the colR-deficient bacteria experience serious glucose-related stress resulting in lysis of a subpopulation of cells [10]. Importantly, cell lysis does not occur on medium with gluconate which is degraded like glucose through Entner-Doudoroff pathway. To test whether inactivation of the TtgABC efflux pump would affect phenol stress only on glucose or it would have a more general role in phenol tolerance, the growth of newly constructed $\operatorname{tg} B$ - and $\operatorname{tg} C$-deficient strains were examined both on glucose and gluconate minimal media supplemented with different concentrations of phenol (Fig. 1). In accordance with the transposon mutagenesis screen, the disruption of the $\operatorname{tg} A B C$ operon made $P$. putida colR-deficient cells more resistant to phenol, and this behaviour was observed on both, glucose and gluconate medium. However, since the $\operatorname{tg} B$ - and $\operatorname{ttg} C$-deficiency enhanced phenol tolerance also in the wild-type background (Fig. 1), we consider that the
TtgABC efflux pump is related to a general tolerance of bacteria to phenol rather than to a particular phenotype of the colR mutant.

\section{Increased phenol tolerance per se does not alleviate the phenol-enhanced autolysis of glucose-grown colR- deficient cells neither does it restore transposition of Tn4652 in the colR mutant}

In our previous study we showed that phenotypes of the colR-deficient bacteria such as membrane leakiness and cell lysis, which are related with growth on glucose, became more prominent if phenol was added to the medium [10]. For instance, glucose-induced release of cytoplasmic $\beta$-galactosidase into the growth medium due to the autolysis of the colR mutant was significantly enhanced if phenol was supplied [10]. In order to find out whether the increased phenol tolerance can alleviate glucose-induced and phenol-enhanced autolysis of the colRdeficient strain, the $\operatorname{ttg} C$-knockout derivatives were subjected to $\beta$-galactosidase assay. To calculate the percentage of unmasked $\beta$-galactosidase activity which was used as an indicator of membrane leakiness and cell lysis, the enzyme activity was measured both in suspension of cells permeabilized with SDS and chloroform (total activity), and in that of intact, non-permeabilized cells. In accordance with our previous results only $4 \%$ of total $\beta$-galactosidase activity was measurable using nonpermeabilized wild-type cells regardless of the presence of phenol in the growth medium [10] (Fig. 2). At the same time, about $15 \%$ of total $\beta$-galactosidase activity was detectable in case of the colR-deficient cells grown on glucose minimal plates, and up to $30 \%$ when cells were 
grown on glucose medium supplemented with $1 \mathrm{mM}$ phenol [10] (Fig. 2). The phenol tolerant $\operatorname{ttg} C$ single mutant behaved in this test like the wild-type strain (Fig. $2)$. We expected that enhanced phenol tolerance will reduce the effect of phenol on membrane leakiness and autolysis of the colR-deficient strain. However, the colRt$\operatorname{tg} C$ double mutant behaved exactly like its parental colR mutant strain in the $\beta$-galactosidase assay (Fig. 2). Thus, these data show that increased phenol tolerance of the colR-deficient strain acquired by inactivation of TtgABC efflux pump cannot alleviate the effect of phenol as a facilitator of glucose-dependent autolysis.

We have previously shown that transposition of Tn4652 is inhibited in starving colR-deficient strain when $2.5 \mathrm{mM}$ phenol is used to select transposon insertion mutants that have gained the ability to grow on phenol [9]. Yet, if lower phenol concentrations were used, transposition of Tn4652 was somewhat recovered [8]. Therefore, we proposed that increased phenol susceptibility would cause inhibition of transposition of Tn4652 in the starving colRdeficient bacteria [8]. To test this possibility we analysed the phenol tolerant $\operatorname{ttgC}$-knockout derivative of the $\operatorname{col} R$ mutant in a transposition assay. The transposition assay of the colRttgC double mutant showed that despite its high phenol tolerance, transposition was still inhibited like in the colR single mutant (data not shown). Therefore, neither the hindrance of transposition nor the glucose-caused cell lysis phenotype of the colR mutant correlated with phenol tolerance of cells.

\section{Survival of the colR and $\operatorname{ttg} C$ mutants in condition of sudden phenol shock resembles that of the wild-type $P$. putida}

Our previous study suggested that the colR-deficient strain is more sensitive to elevated phenol concentrations due to altered membrane permeability [8]. Propidium iodide staining of glucose-grown bacteria evidenced that a subpopulation of the colR mutant possesses indeed highly permeable membrane [10]. In order to clarify whether elevated phenol entrance could cause the lowered phenol tolerance of the colR mutant we measured the viability of bacteria that were exposed to high phenol concentration over a short time period. We expected that if phenol entry into the colR mutant is increased then the cells of the colR-deficient strain should die faster than wild-type cells. Contrary to that, we expected that treatment of the $\operatorname{ttg} C$ mutant with toxic concentration of phenol will demonstrate long-lasting tolerance of this strain to the toxicant. Unexpectedly, the colony forming ability of bacteria (approximately $10^{8}$ cells $/ \mathrm{ml}$ ) exposed to 50 $\mathrm{mM}$ phenol did not depend on the functionality of the ColRS system nor the TtgABC efflux pump. After 10 minutes about $70 \%$ of the cells were alive independent of their genetic background. By 20 minutes more than $99 \%$ of $P$. putida wild-type as well as of colR-, ttgC- and colRt$\operatorname{tg} C$-deficient cells were dead (not able to form colonies on selective media) and after 30 minutes of treatment with $50 \mathrm{mM}$ phenol the count of viable cells of all strains had dropped by four orders of magnitude. This data suggests that the cell membrane of the colR-deficient strain is not more permeable to phenol than the membrane of the wild-type cells.

\section{ColRS system and TtgABC efflux pump affect phenol tolerance only in growing bacteria}

To further investigate variation in phenol sensitivity between the wild-type, $\operatorname{col} R, \operatorname{ttg} C$ and $\operatorname{col} R \operatorname{ttg} C$ mutant strains we next monitored the 24-hour-viability of bacteria treated with different concentrations of phenol. To evaluate the effect of different physiological conditions, liquid M9 minimal medium contained either $10 \mathrm{mM}$ glucose, $10 \mathrm{mM}$ gluconate or no carbon source at all. As expected, significant differences between the wild-type and colR-deficient strains became evident when phenol tolerance was tested on glucose minimal medium. However, differently from solid glucose medium where $c o l R$ mutant is able to grow at phenol concentration as high as $6 \mathrm{mM}$ (Fig. 1), growth of the colR mutant in liquid glucose medium was restricted already at 2-6 mM phenol concentration. Moreover, whilst the presence of 4-6 mM phenol allowed growth of the wild-type, then the colR mutant started to die at these phenol concentrations and only less than $10 \%$ of inoculated cells could survive during the incubation for 24 hours (Fig. 3A). Another interesting phenomenon detected by us was a specific vulnerability of the glucose-grown colR-deficient strain to intermediate phenol concentrations (4-8 $\mathrm{mM}$ ), which is in contrast with its wild-type-like tolerance to high phenol concentrations (10-16 mM) (Fig. 3A). This data correlates well with our finding that the colR mutant possesses wild-type-like survival in phenol killing assay (see above) and indicates that in totally stressed cells the phenol tolerance is not influenced by ColRS system any more. Analysis of the $\operatorname{tg} \mathrm{C}$ mutants revealed that the effect of the $\operatorname{ttgC}$ disruption on phenol tolerance in the liquid glucose medium was negligible compared to its effect on the solid medium (compare Fig. 1 and 3A). Compared to the wildtype strain, the $\operatorname{tg} C$ mutant tolerated higher phenol concentrations on solid glucose medium (Fig. 1) while in liquid medium there were no differences in phenol tolerance between these two strains (Fig. 3A). Also in the colR-deficient background the effect of $\operatorname{ttg} C$ disruption was stronger on solid than in liquid glucose medium (compare Fig. 1 and 3A). Altogether, these results suggest that in the colR mutant growing in glucose liquid medium glucose stress prevails over phenol stress.

When phenol tolerance was assayed on gluconate liquid medium, the growth and survival of the wild-type and 
colR-deficient strains did not differ at any tested phenol concentration (Fig. 3B). These results diverge from those obtained on solid medium, where $8 \mathrm{mM}$ phenol enabled growth of the wild-type but not that of the colR-mutant (Fig. 1). Thus, in liquid gluconate medium the effect of the colR knockout seems to be less pronounced and is possibly detectable only in a narrow window. Comparison of the $\operatorname{tg} C$-proficient and $t \operatorname{tgC}$-deficient cells revealed clear differences at $8 \mathrm{mM}$ phenol. While the wild-type and colR-deficient strains could not grow at that high phenol concentration and more than $75 \%$ of inoculated cells were killed by 24 hours, the $\operatorname{tg} C$ mutants survived and even grew at $8 \mathrm{mM}$ phenol (Fig. 3B). Thus, deficiency in $\operatorname{tg} C$ increased phenol tolerance of $P$. putida in both liquid and solid gluconate medium.

Surprisingly, in the absence of carbon source, i.e., under growth-restricting conditions, no variations in the viability between the wild-type and the studied mutants were recorded (Fig. 3C). 100\% of inoculated cells of all strains were viable in the presence of $4 \mathrm{mM}$ phenol after 24 hours of incubation (Fig. 3C). The number of viable cells of all strains started to drop by increasing phenol concentration, so that only about $2 \%$ of cells survived at $16 \mathrm{mM}$ phenol (Fig. 3C). The equal phenol tolerance of nongrowing wild-type, colR and $\operatorname{tg} C$ mutants is in clear contrast with their different behaviour under growth-permitting conditions. However, these results are consistent with our data of survival assay with toxic phenol concentration indicating that permeability of their membranes to phenol is similar. Most interestingly, the colR mutant tolerated intermediate phenol concentrations (4-8 mM) in carbon-free medium clearly better than in glucose medium (Fig. 3, compare panels A and C). Thus, presence of glucose remarkably reduces phenol tolerance of colRdeficient strain which obviously occurs due to combination of glucose and phenol stress. Contrary to that, availability of glucose as a carbon and energy source significantly facilitates the tolerance of wild-type $P$. putida to toxic effect of phenol, allowing survival of bacteria at $8 \mathrm{mM}$ phenol, i.e., at concentration which kills majority of starving wild-type bacteria (Fig. 3A and 3C). These data are consistent with earlier publications indicating that several energy-demanding mechanisms contribute to fight against phenol by Pseudomonas cells $[4,32,33]$. Hence, our data show that ColRS system and TtgABC pump are involved in phenol tolerance of $P$. putida only under growth conditions indicating that especially growth-related processes of phenol tolerance are affected by both these systems.

\section{Presence of phenol in growth medium enhances proportion of cells with higher DNA content}

Flow cytometry is a technique which allows to analyse microbial population at single cell level and to detect dis-

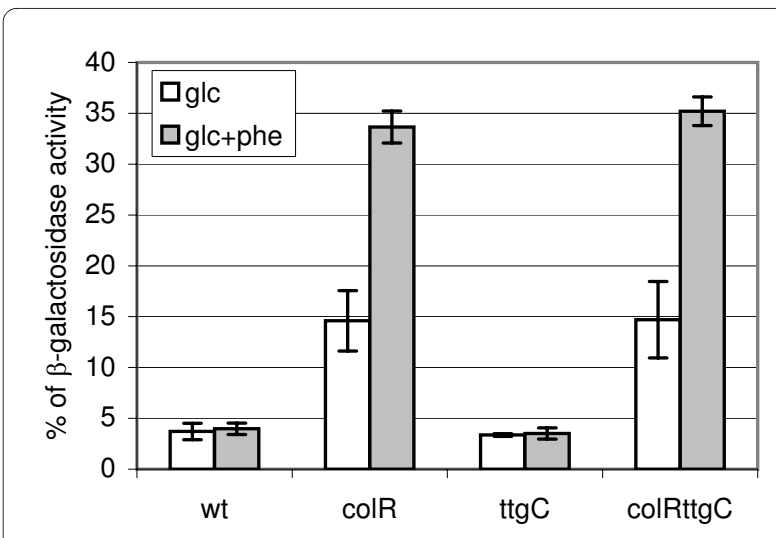

Figure 2 Unmasked $\beta$-galactosidase activity as an indicator of membrane leakiness and cell lysis. The data present percentage of $\beta$-galactosidase activity, measured from non-permeabilized cells against total enzyme activity determined from permeabilized bacteria. Results for P. putida PaW85 (wt), colR-deficient (colR), ttgC-deficient (tt$\mathrm{gC}$ ) and colRttgC double mutant (colRttgC) strains are shown. Bacteria were grown overnight on solid glucose M9 minimal medium ( $\mathrm{glc}$ ) or on the same medium supplemented with $1 \mathrm{mM}$ phenol ( $\mathrm{glc}+\mathrm{phe}$ ). Data (mean \pm standard deviation) of at least three independent determinations are presented.

tinct subpopulations with different functional and structural parameters. We have previously shown that population of solid medium-grown $P$. putida is heterogeneous by its DNA content and membrane permeability to propidium iodide (PI) when analysed with flow cytometry [10]. In order to assess how the wild-type $P$. putida and its $c o l R$ - and $\operatorname{tg} C$-deficient derivatives change their population structure as well as membrane permeability when growing on different media supplemented with phenol, the microbial populations were analysed at single cell level.

Flow cytometry analysis of solid medium-grown bacteria stained with the mixture of SYTO9 and PI demonstrated highly heterogeneous population structure with seven clearly distinguishable subpopulations (Fig. 4). Cells in the first three subpopulations, named as C1, C2 and $\mathrm{C} 3+$, are considered completely healthy as they do not stain with PI. These three populations differ from each other by their SYTO9 fluorescence intensity which most probably reflects their different DNA content. Next three populations, C1_perm, C2_perm and C3+_perm, are considered together as cells with membrane permeable to PI but they can be also distinguished by different DNA content analogous to populations C1, C2 and C3+. This was supported by comparative analysis of SYTO9only and SYTO9+PI-stained populations which revealed that subpopulations $\mathrm{C} 1, \mathrm{C} 2$ and $\mathrm{C} 3+$ observed with SYTO9 alone were equal to the sums of their respective healthy and PI-permeable subpopulations in case of SYTO9 and PI double staining (Additional File 2). Seventh subpopulation, marked as Dead, is clearly present 
only in glucose-grown colR-deficient cells (Fig. 4 and Additional File 3) and correlates with cell lysis. Therefore, this subpopulation most probably represents dead cells with strongly damaged membranes and even lowered DNA content. Latter is supported by observation that glucose-grown colR-deficient cells had subpopulation with remarkably lower green fluorescence when stained with SYTO9 only (Additional File 3). In addition, Dead subpopulation has lower side scatter (SSC) indicating that these cells have less complex intracellular structure compared to other cells (Additional File 3).

Some general observations about the effect of phenol on population structure were made by SYTO9/PI staining and single cell analysis. Most strikingly, independent of $P$. putida strain analysed and carbon source used (glucose or gluconate), addition of phenol to growth medium significantly enhanced proportion of populations $\mathrm{C} 2$ and $\mathrm{C} 3+$, i.e., those with higher DNA content (Fig. 5), indicating that phenol primarily inhibits cell division and not so much DNA replication. Second, in case of all strains and growth conditions phenol enhanced proportion of PI permeable cells but except for the colR-deficient strains grown on glucose this effect was rather modest (Fig. 5). Three PI permeable subpopulations together (C1_perm, C2_perm and C3+_perm) constituted approximately 1$2 \%$ of the population of the wild-type and $\operatorname{ttgC}$-deficient strain when bacteria were grown on glucose medium. If growth medium was supplemented with $3 \mathrm{mM}$ phenol then the relative amount of PI permeable cells raised up to $5 \%$, and in the presence of $8 \mathrm{mM}$ phenol up to $10 \%$ (Fig. $5 \mathrm{~A})$. On gluconate the proportion of PI permeable cells was 3-5\% in all investigated strains. The presence of 6 $\mathrm{mM}$ phenol in gluconate medium increased the relative amount of PI permeable cells up to $15 \%$ and $8 \mathrm{mM}$ phenol up to $16 \%$ (Fig. 5B). Notably, there were more cells with enhanced membrane permeability to PI among populations $\mathrm{C} 2$ and $\mathrm{C} 3+$ (containing cells with higher DNA content) than that in $\mathrm{C} 1$ population (Fig. 5). As $\mathrm{C} 2$ and $\mathrm{C} 3+$

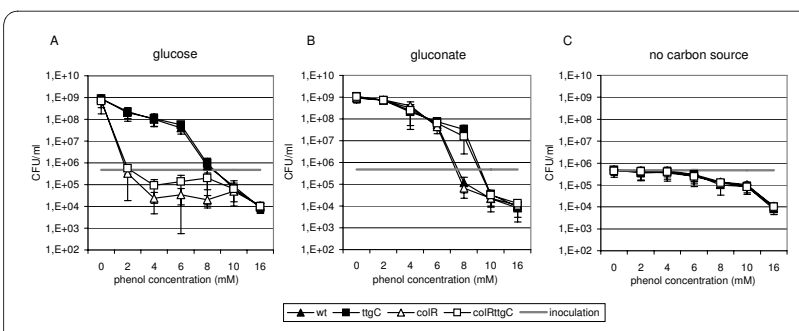

Figure 3 Liquid medium assay of phenol tolerance. CFU of $P$. putida wild-type (wt), colR-deficient (colR), ttgC-deficient (ttgC) and colRtt$g$ Cdouble mutant (colRttgC) strains in the presence of different phenol concentrations. Phenol sensitivity was evaluated in liquid M9 minimal medium in the presence of $10 \mathrm{mM}$ glucose (A) or $10 \mathrm{mM}$ gluconate (B) or in the absence of carbon source (C). Data (mean \pm standard deviation) of at least three independent determinations are presented. cells are those most probably preparing to divide this suggests that temporary enhanced membrane permeability can occur due to cell division.

In accordance with our previous results [10] flow cytometry analysis of the colR mutant revealed high amount of cells with membrane permeable to PI when grown on solid glucose medium (Fig. 5A). The presence of phenol in growth medium significantly enhanced proportion of subpopulations with PI-permeable membrane and furthermore, drastically increased subpopulation of dead cells (Fig. 5A). However, these effects are specific to glucose as they do not occur on gluconate medium (Fig. $5 \mathrm{~B})$. Thus, the results of flow cytometry analysis confirmed that the colR mutant experiences specific membrane leakiness-causing stress only if grown on glucose solid medium and phenol can enhance this phenomenon.

Interestingly, although the wild-type and the colR mutant do not differ from each other in respect of proportion of PI-permeable cells when grown on gluconate medium with $6 \mathrm{mM}$ phenol, they still differ if we compare proportions of subpopulations with different DNA content. The phenol-exposed colR-deficient strain demonstrates higher amount of cells in C3+ subpopulation than that of the wild-type (Fig. 5B, p = 0.02). From enhancement of $\mathrm{C} 3+$ subpopulation with higher DNA content, we concluded, that phenol has stronger cell division-arresting effect on the colR-deficient cells than on the wildtype.

Flow cytometry experiments evidenced that the disruption of $\operatorname{ttg} C$ does not affect cell membrane permeability to PI (Fig. 5). Neither can it affect the proportion of dead cells in the glucose grown colR-mutant which is in good accordance with $\beta$-galactosidase measurements data (Fig. 2 and Fig. 5A). However, the disruption of $\operatorname{ttg} C$ affects ratio of subpopulations with different DNA content. On gluconate medium supplemented with $6 \mathrm{mM}$ phenol the amount of cells with higher DNA content $(\mathrm{C} 3+$ plus C3+_perm) is lower in the colR $\operatorname{ttg} C$ double mutant compared to the colR single mutant (Fig. 5B, $\mathrm{p}=0.027$ ). The effect of $\operatorname{ttg} C$ becomes evident also in the colR proficient background, yet, it occurs at higher phenol concentrations. Compared to the wild-type there are less cells in subpopulations $\mathrm{C} 3+$ and $\mathrm{C} 3+$ _perm of the $\operatorname{ttg} \mathrm{C}$ mutant when cells were grown in the presence of $8 \mathrm{mM}$ phenol on either glucose or gluconate (Fig. $5, p=0.025$ and $p=$ 0.016 , respectively). These results suggest that inactivation of TtgABC efflux pump can alleviate the phenolcaused cell division arrest.

\section{Discussion}

Phenol as chaotropic solute can cause different kind of damage such as increase in a leakiness of membrane, enhance oxidative stress, and destabilize macromolecules due to the reduced water activity [4]. Therefore, there are 
several cellular targets which can be disturbed by phenol. It is known that membrane permeabilizing effect of phenol as well as other aromatic compounds is reduced by rigidification of cell membrane, thus maintaining optimal cell membrane fluidity and permeability [3,34]. Our flow cytometry analysis of phenol-exposed P. putida cultures demonstrated that phenol only slightly increased the amount of cells with PI permeable membrane indicating that cells quite well maintain their membrane homeostasis (Fig. 5). Instead, flow cytometry data indicated that the cell division step of the cell cycle is particularly sensitive to the toxic effect of phenol. This was concluded from the finding that the population structure changed essentially if growth medium contained high concentration of phenol. We observed that phenol caused accumulation of cells with higher DNA content indicating cell division arrest (Fig. 5). Phenol is considered to be toxic primarily because it easily dissolves in membrane compartments of cells, so impairing membrane integrity [35]. Considering that cell division and membrane invagination need active synthesis of membrane components, it is understandable that this step is sensitive to membraneactive toxicant, and in this context, inactivation of cell division is highly adaptive for P. putida exposed to phenol. In accordance with our findings, literature data also suggest that cell division arrest may act as an adaptive mechanism to gain more time to repair phenol-caused membrane damage. For example, it has been shown by proteomic analysis that sub-lethal concentrations of phenol induce cell division inhibitor protein MinD in $P$. putida [32]. It was also shown that cells of different bacterial species became bigger when grown in the presence of membrane-affecting toxicant [36]. Authors suggested that bigger cell size reduces the relative surface of a cell and consequently reduces the attachable surface for toxic aromatic compound [36]. However, our flow cytometry analysis showed that cell size (estimated by forward scatter) among populations with different DNA content $(\mathrm{C} 1$, $\mathrm{C} 2$ and $\mathrm{C} 3+$ ) did not change in response to phenol (data not shown). In all growth conditions the average size of cells with higher DNA content was obviously bigger than the size of cells with lower DNA content (data not shown). Therefore, our data indicate that phenol-caused accumulation of bigger cells occurs due to inhibition of cell division which helps to defend the most sensitive step of cell cycle against phenol toxicity.

In this study we disclosed several genetic factors that influence the phenol tolerance of $P$. putida. The finding that disturbance of intact TtgABC efflux machinery enhances phenol tolerance of $P$. putida is surprising because this pump contributes to toluene tolerance in $P$. putida strain DOT-T1E $[28,37]$. So, our data revealed an opposite effect in case of phenol. In toluene tolerance the effect of TtgABC pump is obvious as it extrudes toluene
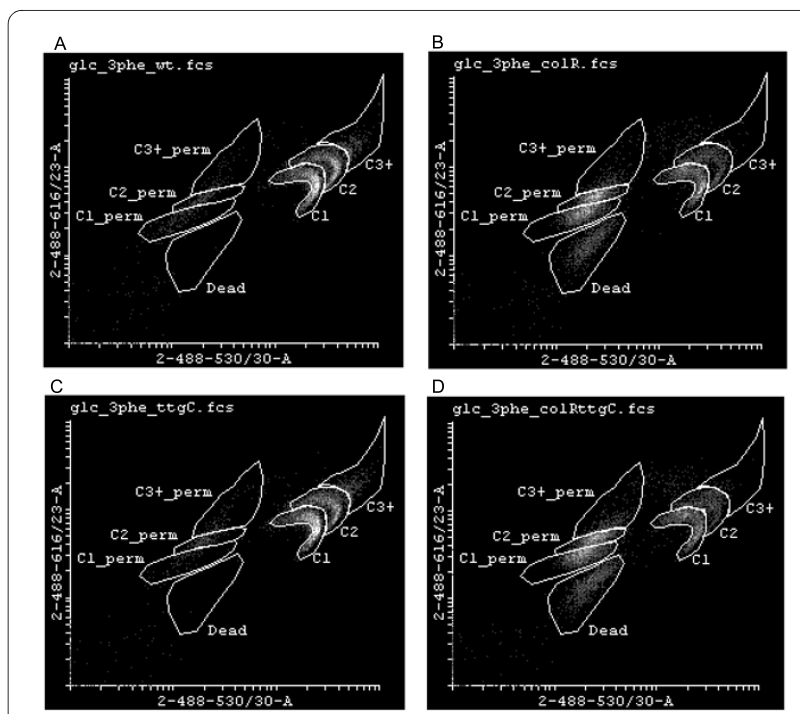

Figure 4 Visualization of subpopulations by flow cytometry analysis. P. putida wild-type (A), colR-deficient (B), ttgC-deficient (C) and colRttgC double mutant (D) strains, grown for $24 \mathrm{~h}$ on glucose minimal plates supplemented with $3 \mathrm{mM}$ phenol were stained using red fluorescence dye propidium iodide (PI) and green fluorescence dye SYTO9, which both stain nucleic acids. Each dot represents an event, analysed by flow cytometer, that has been exicated at $488 \mathrm{~nm}$ and respective fluorescence emission has been measured at 530 (30) and 616 (23) nm. Area of seven different subpopulations is indicated. Density plot of results is presented where lighter areas indicated more events with same parameters.

[28], yet, its negative effect in phenol tolerance is not so easily understandable. Our results excluded the possibility that disruption of TtgABC pump can affect membrane permeability to phenol. Rather, flow cytometry data suggest that functionality of TtgABC pump may somehow affect cell division checkpoint. This is supported by the finding that phenol-exposed population of the $\operatorname{tg} C$ mutant contained relatively less cells with higher DNA content than that of the wild-type, implying that in the $\operatorname{ttg} C$-deficient strain the cell division is less inhibited by phenol than that in the $\operatorname{tg} \mathrm{C}$-proficient strain. Interestingly, the MexAB-OprM pump, the TtgABC ortholog in $P$. aeruginosa, facilitates efflux of a quorum sensing molecule, $N$-(3-oxododecanoyl) homoserine lactone [38]. The TtgABC homologue in Escherichia coli, AcrAB-TolC, is also involved in extrusion of quorum sensing signals and in regulation of population entering into stationary phase. Namely, it has been shown that acrAB-deficient strain can grow to higher cell density in stationary phase than the wild-type E. coli [39] indicating that its cell division is less inhibited by stationary phase factors. In case of $P$. putida, however, we found no evidence that inactivation of TtgABC pump could affect the growth of bacterial culture in stationary phase, as judged by optical density measurements (data not shown). Nevertheless, flow 


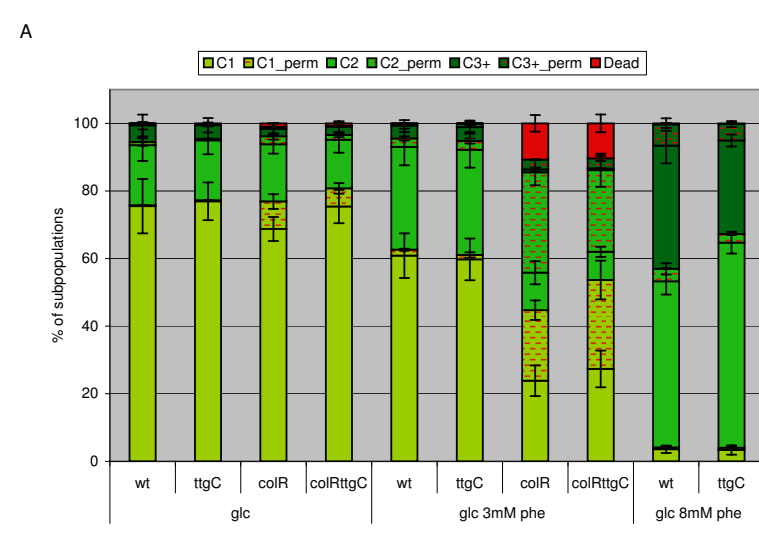

B

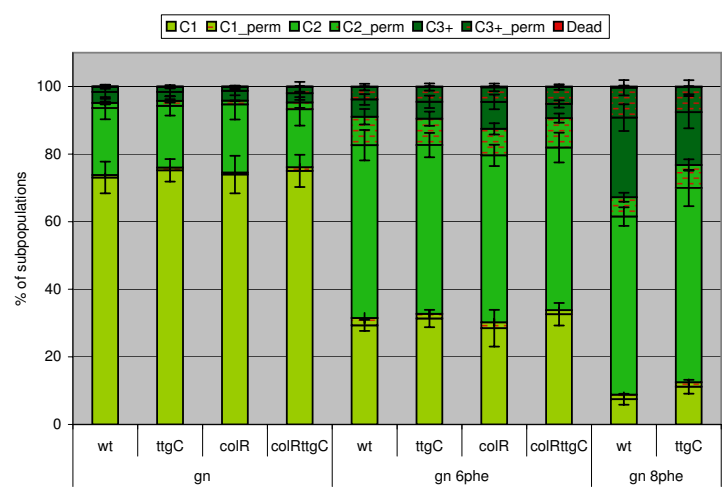

Figure $\mathbf{5}$ Cell population structure by flow cytometry analysis. $P$. putida wild-type (wt), colR-deficient (colR), ttgC-deficient (ttgC) and colRttgC double mutant (colRttgC) strains were grown for $24 \mathrm{~h}$ on glucose (A) or gluconate (B) minimal plates. Concentration of phenol (phe) in growth medium (either $3 \mathrm{mM}, 6 \mathrm{mM}$ or $8 \mathrm{mM}$ ) is indicated below the bars. Cells were stained with PI and SYTO9 and analysed by flow cytometry. Relative proportions of seven subpopulations (as indicated in Figure 4 ) are shown. Data (mean \pm standard deviation) of at least three independent determinations are presented.

cytometry analysis of the phenol-exposed P. putida $\operatorname{ttg} C$ mutant revealed population structure indicative of more active cell division than that of the wild-type. However, at this stage of studies we cannot distinguish whether less arrested cell division is a reason for the increased phenol tolerance of the $\operatorname{ttg} C$ mutant or, vice versa, increased phenol tolerance results in less-inhibited cell division.

In our previous study, where we showed that the colRdeficient $P$. putida is less tolerant to phenol than its parental strain, we argued that membrane permeability of the colR mutant to phenol may be increased [8]. However, results of the current study suggest that the phenol entry into the colR-deficient strain is not increased. The latter was supported by the assay which measured the ability of glucose-grown cells to survive in the presence of $50 \mathrm{mM}$ phenol. Unexpectedly, no differences in cell survival between the wild-type and the colR-deficient strain were recorded after phenol-shock, indicating similar mem- brane permeability to phenol in the colR-deficient and the wild-type cells. As phenol is known to cause membrane permeabilization [40] we therefore tested whether population of phenol-exposed colR-deficient strain could contain more cells with PI permeable membrane. However, as judged by flow cytometry analysis of gluconate-grown bacteria, also the membrane permeabilizing effect of phenol is similar to the wild-type and the colR mutant (Fig. 5). Thus, other reasons than enhanced phenol entry or increased membrane permeability should underlie behind the lowered phenol tolerance of the colR mutant.

Interestingly, population analysis at single cell level revealed that compared to the wild-type, phenol more efficiently enhanced the relative amount of subpopulations with higher DNA content in case of the colR mutant, suggesting that cell division of the colR mutant is more sensitive to phenol inhibition than that of the wildtype (Fig. 5). However, it is hard to distinguish whether it occurs due to lowered phenol tolerance or reflects some sort of specific response. Surprisingly, our current study demonstrates that phenol sensitivity of the colR-deficient strain drastically depends on whether bacteria are growing or not - no effect of the ColRS system on phenol tolerance of $P$. putida could be detected under conditions of starvation (Fig. 3C). Thus, our data imply that state of metabolic dormancy prevents phenol from hitting its target in the colR-deficient cells. We have previously shown that ColR regulates several membrane proteins and is involved in avoidance of several membrane-related disorders $[8,10,12]$. Therefore it is reasonable to suppose that absence of ColR specifically impairs synthesis or turnover of membrane components and this leads to the reduced phenol tolerance in case of actively growing bacteria. However, in starving cells synthesis reactions are downregulated and that may cut off the effect of ColR deficiency on phenol tolerance. Such scenario would also explain why differences in survival between the wild-type and the colR-deficient strain disappear under growthpermitting conditions at elevated phenol concentrations (Fig. 3A). Eventually, high phenol concentration will totally inhibit biosynthetic processes necessary for cell growth and division, thereby eliminating the target of phenol action in the colR mutant.

In addition to increased phenol stress, the colR mutant experiences serious glucose-specific stress resulting in cell lysis [10]. Importantly, the presence of phenol strongly enhances glucose-dependent cell lysis of the colR mutant as well as proportion of cells with PI-permeable membrane (Fig. 3 and 5). This raises an interesting question about interconnections between phenol- and glucose-caused stresses experienced by the colR-deficient $P$. putida. It has been shown by Santos and co-workers that phenol induces expression of proteins involved in cell envelope biosynthesis. Namely, LpxC (UDP-3-O-acyl N- 
acetylglucosamine deacetylase) and MurA (UDP-Nacetylglucosamine enolpyruvyl transferase) are induced by phenol in a concentration-dependent manner [32]. LpxC and MurA are involved in lipopolysaccharide and peptidoglycane biosynthesis, respectively, suggesting that adaptation to phenol involves higher need for synthesis of cell envelope components. As both pathways use UDP-Nacetylglucosamine, this suggests also enhancement of nucleotide sugar metabolism in response to phenol stress. Considering that lysis of the colR-mutant strictly depends on carbon source, the enhancement of glucosedependent cell lysis by phenol could occur through its dual effect on cell metabolism and membrane homeostasis. Our data suggest that although phenol can significantly enhance the glucose-induced stress in case of the colR-deficient strain, nevertheless, the phenol- and glucose-caused stresses are not directly coupled. This was concluded from the cell lysis and membrane permeability measurement data (Fig. 2 and 5) showing that the increased phenol tolerance of the colR-deficient strain acquired by the disruption of the $\operatorname{tg} C$ gene cannot alleviate the effect of phenol as a facilitator of glucose-dependent autolysis of the colR mutant. Our data rather suggest that in the glucose-growing colR-deficient strain phenol can activate or inhibit also signals not directly related to its toxicity. It is possible that some kinds of cell growth or division signals are misread in the presence of phenol in the colR mutant, which eventually leads to the cell lysis. In that case phenol could act as a signal, leading to the cell death, rather than being killing factor itself. Our further experiments will hopefully clarify whether phenoland glucose-caused stresses originate from the same defect of the colR mutant or they are caused by different reasons.

\section{Conclusions}

Current study demonstrates the involvement of the ColRS two-component system and the TtgABC efflux pump in phenol tolerance of $P$. putida. Our results imply that $\operatorname{Ttg} A B C$ and ColRS systems are not directly connected and may affect phenol tolerance via independent pathways. Both these systems affect phenol tolerance of growing cells only but not of starving ones, indicating that ColRS and TtgABC systems affect processes occurring in metabolically active and dividing bacteria. Most tolerance mechanisms to aromatic hydrocarbons are directed toward maintaining the cell membrane intactness [2]. Given that ColRS and TtgABC systems are also implicated in membrane functions $[12,30,38]$, it is reasonable to conclude that they may assist in regulation of biosynthesis and/or turnover of membrane components, so helping to maintain membrane homeostasis during growth and division. Population structure analysis at single cell level revealed that strong cell division inhibition occurred in phenol-exposed population which could be considered as adaptive response to phenol stress to reduce the phenol-caused damage and to maintain membrane homeostasis.

\section{Additional material}

\begin{abstract}
Additional file 1 Plate assay of phenol tolerance of $P$. putida PaW85 (wt) and colR-deficient (colR) strains. Cells were grown on glucose ( $\mathrm{glc}$ ) minimal medium in the presence or absence of $8 \mathrm{mM}$ phenol. Approximate number of inoculated bacterial cells is indicated above the figure. Bacteria were photographed after 4 days of growth.

Additional file $\mathbf{2}$ Comparative analysis of subpopulations with different DNA content by staining of cells with SYTO9 and PI or SYTO9

alone. P. putida wild-type (wt) and ttgC-deficient (ttgC) strains were grown for $24 \mathrm{~h}$ on gluconate minimal plates supplemented with $8 \mathrm{mM}$ phenol. Cells were stained with PI and SYTO9 (SYTO9+PI) or SYTO9 alone and analysed by flow cytometry. Percentage of subpopulations with different DNA content (C1 and C1_perm, C2 and C2_perm, C3+ and C3+_perm) is shown. Data (mean \pm standard deviation) of two independent experiments are presented.

Additional file 3 Description of subpopulation "Dead". P. putida wildtype $(A, C, E)$ and colR-deficient $(B, D, F)$ strains were grown for $24 \mathrm{~h}$ on glucose minimal plates supplemented with $3 \mathrm{mM}$ phenol. Cells were stained with SYTO9 alone (A, B) or with SYTO9 and PI (C-F) and analysed by flow cytometry. Fluorescence at 530 (30) is plotted against fluorescence at 616 (23) $n m(A-D)$ or side scatter of light (SSC-A) (E, F). Fluorescence at 530 (30) measures SYTO9 fluorescence and side scatter of light correlates with size of bacterial cells
\end{abstract}

\section{Authors' contributions}

MP participated in the design of experimental work and manuscript writing. She carried out transposon mutagenesis screen, most phenol tolerance and killing assays, and flow cytometry analysis. $\mathrm{HI}$ constructed mutant strains. LL contributed to the mutagenesis screen and phenol tolerance assays. MK participated in manuscript editing. $\mathrm{RH}$ performed enzyme measurements and coordinated experimental work and manuscript editing. All authors read and approved the final manuscript.

\section{Acknowledgements}

We are grateful to Tiina Alamäe and Paula Ann Kivistik for critically reading the manuscript. We thank Riho Teras for plasmid pUCNotKm. Dimitri Lubenets is specially acknowledged for operating FACSAria. This work was supported by grant 7829 from the Estonian Science Foundation to R. H., and by funding of Targeted Financing Project TLOMR0031 from the Estonian Ministry of Research and Education and by grant HHMI 55005614 from the Howard Hughes Medical Institute International Research Scholars Program to M. K.

\section{Author Details}

Institute of Molecular and Cell Biology, University of Tartu, 51010 Tartu, Estonia

Received: 11 March 2010 Accepted: 14 April 2010

Published: 14 April 2010

\section{References}

1. Dominguez-Cuevas P, Gonzalez-Pastor JE, Marques S, Ramos JL, de Lorenzo V: Transcriptional Tradeoff between Metabolic and Stress response Programs in Pseudomonas putida KT2440 Cells Exposed to Toluene. J Biol Chem 2006, 281(17):11981-11991.

2. Ramos JL, Duque E, Gallegos MT, Godoy P, Ramos-Gonzalez MI, Rojas A, Teran W, Segura A: Mechanisms of solvent tolerance in gram-negative bacteria. Annu Rev Microbiol 2002, 56:743-768.

3. Sikkema J, de Bont JA, Poolman B: Mechanisms of membrane toxicity of hydrocarbons. Microbio/ Rev 1995, 59(2):201-222.

4. Hallsworth JE, Heim S, Timmis KN: Chaotropic solutes cause water stress in Pseudomonas putida. Environ Microbiol 2003, 5(12):1270-1280.

5. Wery J, de Bont JAM: Solvent-tolerance of Pseudomonads: a new degree of freedom in biocatalysis. In Pseudomonas: Biosynthesis of 
macromolecules and molecular metabolism Volume 3. Edited by: Ramos JL. New York: Kluwer Academic/Plenum Publishers; 2004:609-634.

6. Hoch JA, Varughese Kl: Keeping signals straight in phosphorelay signal transduction. J Bacterio/ 2001, 183(17):4941-4949.

7. Dekkers LC, Bloemendaal CJ, de Weger LA, Wijffelman CA, Spaink HP, Lugtenberg $B J$ : A two-component system plays an important role in the root-colonizing ability of Pseudomonas fluorescens strain WCS365. Mol Plant Microbe Interact 1998, 11(1):45-56.

8. Kivistik PA, Putrinš M, Püvi K, Ilves H, Kivisaar M, Hõrak R: The ColRS twocomponent system regulates membrane functions and protects Pseudomonas putida against phenol. J Bacteriol 2006, 188(23):8109-8117.

9. Hõrak R, Ilves H, Pruunsild P, Kuljus M, Kivisaar M: The ColR-ColS twocomponent signal transduction system is involved in regulation of Tn4652 transposition in Pseudomonas putida under starvation conditions. Mol Microbiol 2004, 54(3):795-807.

10. Putrinš M, Ilves H, Kivisaar M, Hõrak R: ColRS two-component system prevents lysis of subpopulation of glucose-grown Pseudomonas putida. Environ Microbiol 2008, 10(10):2886-2893.

11. de Weert S, Dekkers LC, Bitter W, Tuinman S, Wijfjes AH, van Boxtel R, Lugtenberg BJ: The two-component colR/S system of Pseudomonas fluorescens WCS365 plays a role in rhizosphere competence through maintaining the structure and function of the outer membrane. FEMS Microbiol Ecol 2006, 58(2):205-213.

12. Kivistik PA, Kivi R, Kivisaar M, Hõrak R: Identification of ColR binding consensus and prediction of regulon of ColRS two-component system. BMC molecular biology 2009, 10:46.

13. Bayley SA, Duggleby CJ, Worsey MJ, Williams PA, Hardy KG, Broda P: Two modes of loss of the Tol function from Pseudomonas putida mt-2. Mol Gen Genet 1977, 154(2):203-204.

14. Nelson KE, Weinel C, Paulsen IT, Dodson RJ, Hilbert H, Martins dos Santos VA, Fouts DE, Gill SR, Pop M, Holmes M, et al:: Complete genome sequence and comparative analysis of the metabolically versatile Pseudomonas putida KT2440. Environ Microbiol 2002, 4(12):799-808.

15. Carter P, Bedouelle H, Winter G: Improved oligonucleotide site-directed mutagenesis using M13 vectors. Nucleic Acids Res 1985, 13(12):4431-4443.

16. Herrero M, de Lorenzo V, Timmis KN: Transposon vectors containing non-antibiotic resistance selection markers for cloning and stable chromosomal insertion of foreign genes in gram-negative bacteria. $J$ Bacteriol 1990, 172(11):6557-6567.

17. Boyer HW, Roulland-Dussoix D: A complementation analysis of the restriction and modification of DNA in Escherichia coli. J Mol Biol 1969, 41(3):459-472.

18. Figurski DH, Helinski DR: Replication of an origin-containing derivative of plasmid RK2 dependent on a plasmid function provided in trans. Proc Natl Acad Sci USA 1979, 76(4):1648-1652.

19. Miller $\mathrm{JH}$ : A short course in bacterial genetics: a laboratory manual and handbook for Echerichia coli and related bacteria. Cold Spring Harbour Laboratory Press, Cold Spring Harbour, NY; 1992.

20. Adams MH: Bacteriophages. Intersciensce Publishers Inc., NY; 1959

21. Wilson KJ, Sessitsch A, Corbo JC, Giller KE, Akkermans AD, Jefferson RA: beta-Glucuronidase (GUS) transposons for ecological and genetic studies of rhizobia and other gram-negative bacteria. Microbiology 1995, 141(Pt 7):1691-1705

22. O'Toole GA, Kolter R: Initiation of biofilm formation in Pseudomonas fluorescens WCS365 proceeds via multiple, convergent signalling pathways: a genetic analysis. Mol Microbiol 1998, 28(3):449-461.

23. de Lorenzo V, Herrero M, Jakubzik U, Timmis KN: Mini-Tn5 transposon derivatives for insertion mutagenesis, promoter probing, and chromosomal insertion of cloned DNA in gram-negative eubacteria. $J$ Bacteriol 1990, 172(11):6568-6572.

24. Pavel H, Forsman M, Shingler V: An aromatic effector specificity mutant of the transcriptional regulator DmpR overcomes the growth constraints of Pseudomonas sp. strain CF600 on para-substituted methylphenols. J Bacteriol 1994, 176(24):7550-7557.

25. Hõrak R, Kivisaar M: Expression of the transposase gene tnpA of Tn4652 is positively affected by integration host factor. J Bacteriol 1998, 180(11):2822-2829.

26. Tover A, Ojangu EL, Kivisaar M: Growth medium compositiondetermined regulatory mechanisms are superimposed on CatR- mediated transcription from the pheBA and catBCA promoters in Pseudomonas putida. Microbiology 2001, 147(Pt 8):2149-2156.

27. Stocks SM: Mechanism and use of the commercially available viability stain, BacLight. Cytometry A 2004, 61(2):189-195.

28. Rojas A, Duque E, Mosqueda G, Golden G, Hurtado A, Ramos JL, Segura A: Three efflux pumps are required to provide efficient tolerance to toluene in Pseudomonas putida DOT-T1E. J Bacteriol 2001, 183(13):3967-3973.

29. Duque E, Segura A, Mosqueda G, Ramos JL: Global and cognate regulators control the expression of the organic solvent efflux pumps TtgABC and TtgDEF of Pseudomonas putida. Mol Microbiol 2001, 39(4):1100-1106.

30. Teran W, Felipe A, Segura A, Rojas A, Ramos JL, Gallegos MT: Antibioticdependent induction of Pseudomonas putida DOT-T1E TtgABC efflux pump is mediated by the drug binding repressor TtgR. Antimicrob Agents Chemother 2003, 47(10):3067-3072.

31. Teran W, Krell T, Ramos JL, Gallegos MT: Effector-Repressor Interactions, Binding of a Single Effector Molecule to the Operator-bound TtgR Homodimer Mediates Derepression. J Biol Chem 2006, 281(11):7102-7109

32. Santos PM, Benndorf D, Sa-Correia I: Insights into Pseudomonas putida KT2440 response to phenol-induced stress by quantitative proteomics. Proteomics 2004, 4(9):2640-2652.

33. Santos PM, Roma V, Benndorf D, von Bergen M, Harms H, Sa-Correia I: Mechanistic insights into the global response to phenol in the phenolbiodegrading strain Pseudomonas sp. M1 revealed by quantitative proteomics. Omics 2007, 11(3):233-251.

34. Heipieper HJ, de Bont JA: Adaptation of Pseudomonas putida S12 to ethanol and toluene at the level of fatty acid composition of membranes. Appl Environ Microbiol 1994, 60(12):4440-4444.

35. Denich TJ, Beaudette LA, Lee H, Trevors JT: Effect of selected environmental and physico-chemical factors on bacterial cytoplasmic membranes. Journal of microbiological methods 2003, 52(2):149-182.

36. Neumann G, Veeranagouda Y, Karegoudar TB, Sahin O, Mausezahl I, Kabelitz N, Kappelmeyer U, Heipieper HJ: Cells of Pseudomonas putida and Enterobacter sp. adapt to toxic organic compounds by increasing their size. Extremophiles 2005, 9(2):163-168.

37. Ramos JL, Duque E, Godoy P, Segura A: Efflux pumps involved in toluene tolerance in Pseudomonas putida DOT-T1E. J Bacterio/ 1998, 180(13):3323-3329.

38. Pearson JP, Van Delden C, Iglewski BH: Active efflux and diffusion are involved in transport of Pseudomonas aeruginosa cell-to-cell signals. $J$ Bacteriol 1999, 181(4):1203-1210

39. Yang S, Lopez CR, Zechiedrich EL: Quorum sensing and multidrug transporters in Escherichia coli. Proc Natl Acad Sci USA 2006, 103(7):2386-2391.

40. Heipieper HJ, Keweloh H, Rehm HJ: Influence of phenols on growth and membrane permeability of free and immobilized Escherichia coli. Appl Environ Microbiol 1991, 57(4):1213-1217.

doi: $10.1186 / 1471-2180-10-110$

Cite this article as: Putrinš et al., The impact of ColRS two-component system and TtgABC efflux pump on phenol tolerance of Pseudomonas putida becomes evident only in growing bacteria BMC Microbiology 2010, 10:110

\section{Submit your next manuscript to BioMed Central and take full advantage of:}

- Convenient online submission

- Thorough peer review

- No space constraints or color figure charges

- Immediate publication on acceptance

- Inclusion in PubMed, CAS, Scopus and Google Scholar

- Research which is freely available for redistribution 